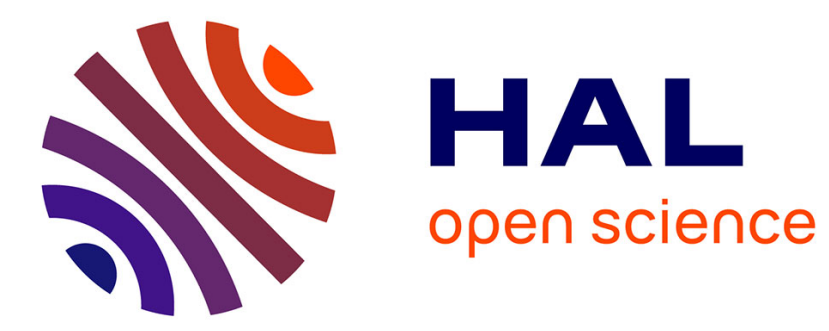

\title{
A New Robust Approach for Highway Traffic Density Estimation
}

Fabio Morbidi, Luis Leon Ojeda, Carlos Canudas de Wit, Iker Bellicot

\section{To cite this version:}

Fabio Morbidi, Luis Leon Ojeda, Carlos Canudas de Wit, Iker Bellicot. A New Robust Approach for Highway Traffic Density Estimation. ECC 2014 - 13th European Control Conference, Jun 2014, Strasbourg, France. pp.2576-2580. hal-00979684

\section{HAL Id: hal-00979684 https://hal.science/hal-00979684}

Submitted on 16 Apr 2014

HAL is a multi-disciplinary open access archive for the deposit and dissemination of scientific research documents, whether they are published or not. The documents may come from teaching and research institutions in France or abroad, or from public or private research centers.
L'archive ouverte pluridisciplinaire HAL, est destinée au dépôt et à la diffusion de documents scientifiques de niveau recherche, publiés ou non, émanant des établissements d'enseignement et de recherche français ou étrangers, des laboratoires publics ou privés. 


\title{
A New Robust Approach for Highway Traffic Density Estimation
}

\author{
Fabio Morbidi, Luis León Ojeda, Carlos Canudas de Wit, Iker Bellicot
}

\begin{abstract}
In this paper we present a robust mode selector for the uncertain graph-constrained Switching Mode Model (SMM), which we use to describe the highway traffic density evolution. Assuming an uncertain speed of the congestion wave, the proposed selector relies on a transition digraph suitably incorporating the present and historical statistical traffic information, to determine the most probable current mode of the SMM. Its effectiveness is demonstrated on the problem of traffic density reconstruction via a switching observer, in an instrumented $2.2 \mathrm{~km}$ highway section of Grenoble south ring in France.
\end{abstract}

\section{INTRODUCTION}

\section{A. Motivation and related work}

An informative parameter for describing the level of congestion in a highway is the traffic density, i.e. the number of vehicles per kilometer. Unfortunately, there do not exist, at present, practical and inexpensive ways to measure this parameter on the field: one should then resort to indirect measurements, such as vehicle flows and speeds, and to suitable dynamic traffic models. The first continuous highway model was proposed by Lighthill, Whitham and Richards in the '50s (the LWR model), and leverages the so-called "fundamental diagram", an empirical curve relating observed vehicle densities to observed flows at a particular road location. More recently, the Cell Transmission Model (CTM) [1] and the related Switching Mode Model (SMM) [2] have attracted considerable attention in the literature: the SMM is a piecewise-affine state-dependent discrete-time system based on the CTM which is well suited for model-based traffic estimation [2], [3] and control [4], [5].

One of the major obstacles to reliable management of real traffic systems is the presence of large modeling uncertainties such as, e.g., uncertainties in the parameters of the fundamental diagram and in the demand and supply functions. In order to address this issue, a considerable effort has been recently devoted towards the design of robust algorithms for traffic density estimation.

The existing algorithms have primarily dealt with parametric uncertainties and can be classified into two main categories: $i$ ) deterministic approaches, in which an interval or set representation is adopted for the uncertainties, and ii) stochastic approaches in which the uncertainties are treated as random variables/processes with known probability distributions. The approach described in [6] belongs to the first category. Here, the authors present a CTM-based setvalued estimator and provide guaranteed bounds on traffic density evolution. The fundamental diagram is assumed to be uncertain (i.e. the cell capacity and jam density vary within

F. Morbidi, L. León Ojeda and I. Bellicot are with the NeCS team, Inria Grenoble Rhône-Alpes, 38334 Montbonnot Saint Martin, France, fabio.morbidi@inria.fr, luis.leon@inria.fr, iker.bellicoteinria.fr.

C. Canudas de Wit is with the NeCS team, GIPSA-lab, UMR-CNRS 5216 , Grenoble, France, carlos . canudas-de-wit@gipsa-lab. fr

This research has been supported by the French project "MOCoPo" through the PREDIT, and by the EU FP7/2010-14 HYCON2 NoE under grant agreement no. 257462. given bounds), and the demand at the origin cells is not perfectly known. The approach in [6] is general, systematic and relatively simple to use. However, it depends on the tuning of a large set of parameters, vehicle densities cannot be reconstructed when traffic measurements are not available at cell boundaries, and it is unclear how conservative are the computed density bounds when the system is not in free flow. A related line of research [7] has explored the use of setvalued fundamental diagrams to more reliably capture the behavior of traffic in the congested regime. Finally, in [8], a distributed approach has been proposed for determining fuzzy confidence intervals for traffic density. However, this heuristic method relies on the identification of a significant number of parameters from the traffic measurements.

A larger body of literature is available on stochastic approaches for robust traffic estimation. In [9], a parameteradaptive filtering approach based on the extended Kalman filter (EKF) has been proposed for the METANET model. The uncertain parameters are determined online by incorporating them into the state vector. Noisy flow and speed measurements at the boundary of two adjacent cells and on/off-ramp flow measurements are used in the correction step of the filter. Although the approach in [9] avoids a timeconsuming offline calibration step and automatically adapts the parameters according to changing external conditions, neither an observability analysis is conducted nor a priori guarantees on the stability of the EKF are given by the authors. Recently, in [10], the adaptive Kalman filtering approach proposed in [9] has been tailored to fit the CTM (inheriting the same pros and cons), and in [11] it has been compared with the unscented Kalman filter for joint and dual estimation. A particle filter is designed in [12] to estimate both speed and traffic density. The particle filter performs well with a small number of particles in light traffic conditions, but obtaining accurate estimates in the presence of severe congestion turns out to be computationally demanding. Finally, in [13], the authors have developed the so-called Stochastic Cell Transmission Model (SCTM), which extends the CTM by considering uncertainties in both the demand and supply functions. In particular, the SCTM defines the free-flow speed, jam-density and congestion-wave speed explicitly as random variables. However, it relies on an oversimplification of the modes of the SMM and it assumes a Gaussian distribution for the random parameters of the fundamental diagram which may be not well explained by physical data.

\section{B. Original contributions and organization}

In this paper, we consider the graph-constrained version of the SMM recently proposed in [3], and introduce an original strategy for robust mode selection. This strategy draws some inspiration from the smooth switching method presented in [5] for ramp metering, and it can be used, in principle, to robustify any SMM-based traffic density estimation algorithm. Assuming an uncertain speed for the congestion wave, 
we incorporate the currently-available information and the statistical information by historical record into a suitable transition digraph or automaton, which supports us in the selection of the most probable current mode of the system. The effectiveness and robustness of the proposed mode selector is demonstrated on the problem of traffic density reconstruction via a switching observer, in a $2.2 \mathrm{~km}$ highway section of Grenoble south ring for which real-time flow and mean speed measurements are available through the "Grenoble Traffic Lab" (GTL), http://necs.inrialpes.fr

The rest of this paper is organized as follows. Sect. II presents our macroscopic traffic model. The robust mode selector is described in Sect. III and its application to the traffic-density reconstruction problem is detailed in Sect. IV. In Sect. V, the main contributions of the paper are summarized and possible future research directions are outlined.

\section{MACROSCOPIC TRAFFIC MODEL}

The traffic behavior is described in this paper by the modified version of the Cell Transmission Model (CTM) introduced in [2]. In this model, the density of a cell evolves according to the conservation law of vehicles, i.e.,

$$
\rho_{i}(k+1)=\rho_{i}(k)+\frac{T}{L_{i}}\left(\varphi_{i}(k)-\varphi_{i+1}(k)\right),
$$

where $\rho_{i}(k)$ is the density of cell $i$ in the road link at time $k \in \mathbb{Z}_{\geq 0}, \varphi_{i}(k)$ is the flow between cell $i-1$ and cell $i$ at time $k, T$ is the discrete-time step and $L_{i}$ the length of cell $i$ where $i \in\{1, \ldots, n\}$ (see Fig. 1). By introducing the demand and supply functions,

$$
\begin{aligned}
D_{i-1}(k) & \triangleq \min \left\{v_{i-1} \rho_{i-1}(k), \varphi_{m, i-1}\right\}, \\
S_{i}(k) & \triangleq \min \left\{\varphi_{m, i}, w_{i}\left(\rho_{m, i}-\rho_{i}(k)\right)\right\},
\end{aligned}
$$

the interface flow $\varphi_{i}(k)$ can be computed as,

$$
\varphi_{i}(k)=\min \left\{D_{i-1}(k), S_{i}(k)\right\},
$$

where $v_{i}$ and $w_{i}$ are respectively the free-flow speed and the speed of the congestion wave in cell $i, \varphi_{m, i}$ is the maximum flow allowed by the capacity of cell $i$, and $\rho_{m, i}$ is the jam density (we omitted the effect of possible on-/offramp flows in (1), which however will appear in Sect. IV-B, in order to keep the presentation simple). For system (1) to be stable, $T$ must satisfy the Courant-Friedrichs-Lewy condition $T \leq \min _{i \in\{1, \ldots, n\}} L_{i} / v_{i}$. Note that according to Fig. 1, $\varphi_{u}=\varphi_{1}$ and $\varphi_{d}=\varphi_{n+1}$, which are referred to as the upstream and downstream flows, respectively, and overall as boundary flows.

Definition 1 (Free and congested cells): The cell $i \in$ $\{1, \ldots, n\}$, is said free and denoted " $F$ " if $\rho_{i} \leq \rho_{c, i}$ where $\rho_{c, i}$ is the critical density of that cell. Otherwise, if $\rho_{c, i}<$ $\rho_{i} \leq \rho_{m, i}$ the cell is said congested and denoted "C". $\diamond$

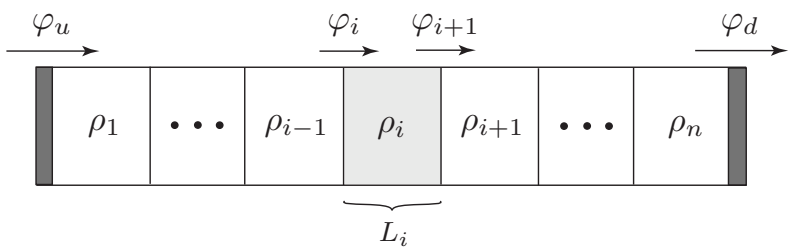

Fig. 1. In the CTM, a road link is subdivided into $n$ cells of length $L_{1}$, $\ldots, L_{n}$ with densities $\rho_{1}, \ldots, \rho_{n}$.
Definition 2 (Ascendant and descendant flows): The cell interface flow $\varphi_{i}, i \in\{1, \ldots, n+1\}$, is said to be ascendant if $\varphi_{i}=S_{i}$ and descendant if $\varphi_{i}=D_{i-1}$. In other words, ascendant “ $\leftarrow$ " (descendant “ $\rightarrow$ ") flows describe waves traveling upwards (downwards), through the interface. $\diamond$

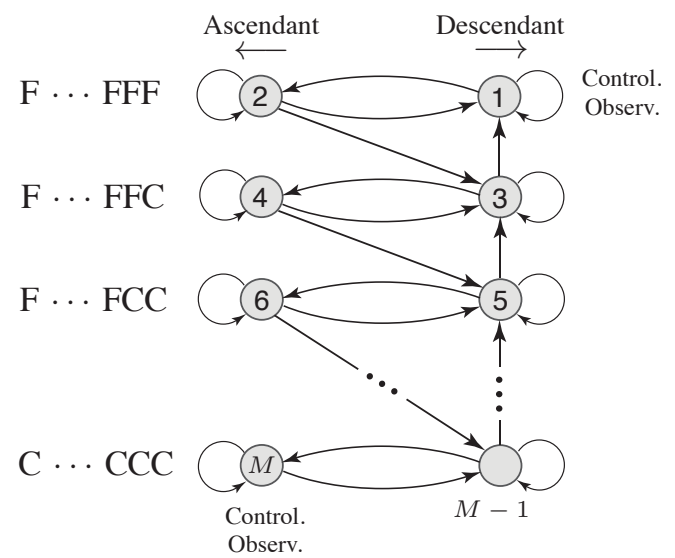

Fig. 2. Digraph $\mathscr{D}_{n}$ of the admissible mode transitions of the SMM.

In order to avoid the nonlinearities present in (1)-(3), we will deal with the Switching Mode Model (SMM) in the graph-constrained version recently proposed in [3]. The $\mathrm{SMM}$ is a piecewise-affine state-dependent system with $M=$ $2(n+1)$ admissible modes, which switches among different sets of linear difference equations depending on the position of the congestion front in the road link and on the transition digraph $\mathscr{D}_{n}$. The nodes in the left side of $\mathscr{D}_{n}$ (see Fig. 2) are relative to the ascendant flows and the nodes in the right side to the descendant flows: by convention mode 1 corresponds to $\overrightarrow{\mathrm{F} \cdots \mathrm{FF}}$, mode 2 to $\overleftrightarrow{\mathrm{F} \cdots \mathrm{FF}}, \ldots$, mode $M$ to $\overleftarrow{\mathrm{C} \cdots \mathrm{CC}}$. We will drop the top arrow when irrelevant to specify whether a mode is ascendant or descendant. Note that the SMM relies on the assumption that there exists only one congestion wave in the road link that appears at cell $n$ and propagates upstream. The graph-constrained SMM admits the following compact state-space representation,

$$
\left\{\begin{array}{l}
\boldsymbol{\rho}(k+1)=\mathbf{A}_{s(k)} \boldsymbol{\rho}(k)+\mathbf{B}_{s(k)} \boldsymbol{\varphi}(k)+\mathbf{E}_{s(k)} \boldsymbol{\rho}_{m}, \\
s(k)=\Sigma\left(\boldsymbol{\rho}(k), \boldsymbol{\varphi}(k), \mathscr{D}_{n}\right) \\
y(k)=h(\boldsymbol{\rho}(k), s(k))
\end{array}\right.
$$

where $\boldsymbol{\rho}=\left[\rho_{1}, \ldots, \rho_{n}\right]^{T}$ is the state vector of cell densities, $\boldsymbol{\varphi}=\left[\varphi_{u}, \varphi_{d}\right]^{T}$ the input, $\boldsymbol{\rho}_{m}=\left[\rho_{m, 1}, \ldots, \rho_{m, n}\right]^{T}$, and

$$
h(\boldsymbol{\rho}(k), s(k))= \begin{cases}\mathbf{C}_{1} \boldsymbol{\rho}(k) & \text { if } s(k)=1, \\ \mathbf{C}_{M} \boldsymbol{\rho}(k)+w_{1} \rho_{m, 1} & \text { if } s(k)=M, \\ 0 & \text { otherwise }\end{cases}
$$

being $\mathbf{C}_{1}=\left[0, \ldots, 0, v_{n}\right]$ and $\mathbf{C}_{M}=\left[-w_{1}, 0, \ldots, 0\right]$. The mode selector $\Sigma\left(\boldsymbol{\rho}(k), \boldsymbol{\varphi}(k), \mathscr{D}_{n}\right)$, which outputs the scalar $s(k) \in\{1, \ldots, M\}$, determines the current mode of the system according to the state and input vectors, and the digraph $\mathscr{D}_{n}$. Note that only modes 1 and $M$ of system (4) are both controllable and observable [3], and that $\mathbf{A}_{2 i+1}=\mathbf{A}_{2 i}$, $\mathbf{B}_{2 i+1}=\mathbf{B}_{2 i}, \mathbf{E}_{2 i+1}=\mathbf{E}_{2 i}, i \in\{1, \ldots, n\}$. The explicit expression of matrices $\mathbf{A}_{s}, \mathbf{B}_{s}$ and $\mathbf{E}_{s}$ can be found in [14], the main differences being the total number of modes $M$, their indexing, and the associated digraph $\mathscr{D}_{n}$. 


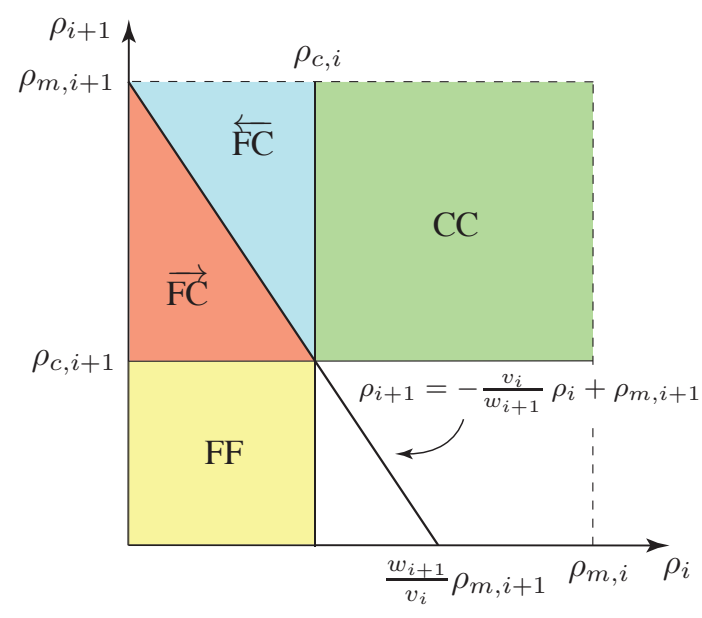

(a)

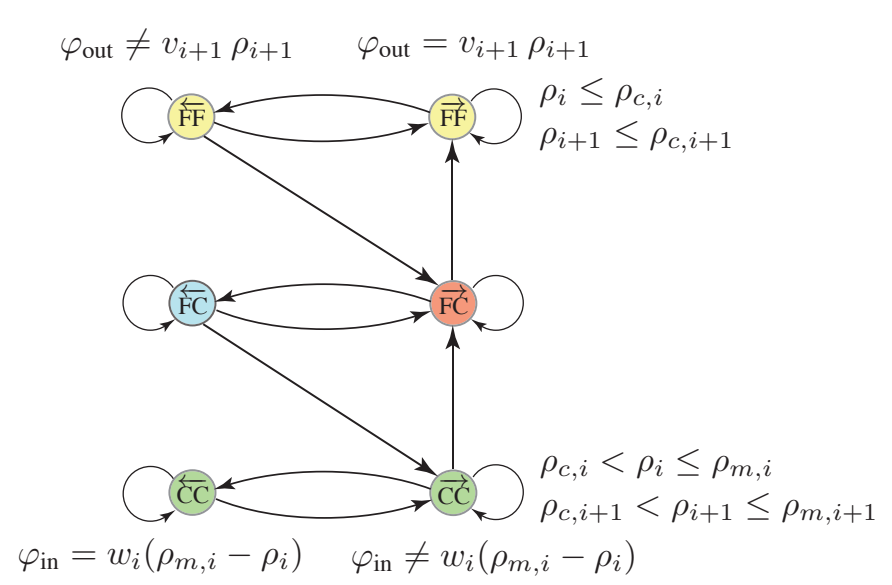

(b)

Fig. 3. Ideal case: (a) Four admissible regions (colored) can be identified in the $\rho_{i} \rho_{i+1}$-plane; (b) Corresponding mode-transition digraph $\mathscr{D}_{2}$ (the switching conditions for the central modes have been omitted for improving readability).

In the following, we will assume that all parameters of system (4) are perfectly known, except for the speed of the congestion wave $w_{i}$. In fact, it is well known that in the fundamental diagram, $v_{i}$ can be estimated fairly accurately from the available flow and speed measurements and $\rho_{m, i}$ can be determined from simple considerations on the road geometry and vehicles' average length (in this paper we assume that the average length of the vehicles, including the inter-vehicular distance, is 8 meters).

\section{ROBUST MODE SELECTOR}

Note that because of the constraints imposed by the transition digraph $\mathscr{D}_{n}$, in our subsequent analysis we can restrict ourselves to the two cells in correspondence to the traffic congestion front, which are henceforth referred to as cell $i$ and cell $i+1$. In what follows, we shall proceed in steps and first analyze the ideal case (i.e. all parameters of system (4) are perfectly known), and then deal with the uncertain case in which the speed $w_{i}$ is not exactly known. A graphical representation of the modes in the density plane $\rho_{i} \rho_{i+1}$ will help us to visualize the admissible regions through which system (4) should transition.

\section{A. Ideal case}

In order to simplify the presentation, let us here assume that the parameters of cells $i$ and $i+1$ are identical and perfectly known, i.e., $v_{i}=v_{i+1}, w_{i}=w_{i+1}$ and $\rho_{m, i}=$ $\rho_{m, i+1}$. We can then identify four admissible regions in the $\rho_{i} \rho_{i+1}$-plane (see Fig. 3(a)):

$\mathrm{FF}: 0<\rho_{i} \leq \rho_{c, i}$ and $0<\rho_{i+1} \leq \rho_{c, i+1}$

$\overrightarrow{\mathrm{FC}}: \rho_{c, i+1}<\rho_{i+1} \leq-\frac{v_{i}}{w_{i+1}} \rho_{i}+\rho_{m, i+1}$

$\overleftarrow{\mathrm{FC}}: 0<\rho_{i} \leq \rho_{c, i}$ and $\rho_{m, i+1} \geq \rho_{i+1}>-\frac{v_{i}}{w_{i+1}} \rho_{i}+\rho_{m, i+1}$

$\mathrm{CC}: \rho_{c, i}<\rho_{i} \leq \rho_{m, i}$ and $\rho_{c, i+1}<\rho_{i+1} \leq \rho_{m, i+1}$

Note that the lower-right white rectangle in Fig. 3(a), i.e. mode CF, is not admissible by hypothesis (cf. Sect. II). Fig. 3(b) shows the restriction of digraph $\mathscr{D}_{n}$ to the twocell case studied in this section: here $\varphi_{\text {in }}$ and $\varphi_{\text {out }}$, refer to the flows entering cell $i$ and exiting cell $i+1$, respectively.

\section{B. Uncertain case}

Let us now assume that the speed of the congestion wave is not exactly known, i.e., $w_{i}=w_{i}^{\text {nom }}+\xi_{i}$, where $w_{i}^{\text {nom }}$ denotes the nominal speed and $\xi_{i}$ is the associated uncertainty. Let us also define (see Fig. 4), $w_{i}^{\min } \triangleq$ $w_{i}^{\text {nom }}-\max _{j \in\{1, \ldots, \ell\}}\left|w_{i, j}-w_{i}^{\text {nom }}\right|, w_{i}^{\max } \triangleq w_{i}^{\text {nom }}+$ $\max _{j \in\{1, \ldots, \ell\}}\left|w_{i, j}-w_{i}^{\text {nom }}\right|$, being $\left\{w_{i, j}\right\}_{j=1}^{\ell}$ a collection of known historical values for the speed of the congestion wave. We can then introduce:

$$
\rho_{c, i}^{\min }=\frac{w_{i}^{\max }}{v_{i}+w_{i}^{\max }} \rho_{m, i}, \quad \rho_{c, i}^{\max }=\frac{w_{i}^{\min }}{v_{i}+w_{i}^{\min }} \rho_{m, i} .
$$

As before, let us assume that $v_{i}=v_{i+1}, \rho_{m, i}=\rho_{m, i+1}$, $w_{i}^{\min }=w_{i+1}^{\min }, w_{i}^{\max }=w_{i+1}^{\max }$, i.e., the parameters of cell $i$ and $i+1$ are identical. We can then identify four admissible deterministic regions in the $\rho_{i} \rho_{i+1}$-plane (colored in Fig. 5):

$$
\begin{array}{ll}
\mathrm{FF}: & 0<\rho_{i} \leq \rho_{c, i}^{\min } \text { and } 0<\rho_{i+1} \leq \rho_{c, i+1}^{\min } \\
\overrightarrow{\mathrm{FC}}: & \rho_{c, i+1}^{\max }<\rho_{i+1} \leq-\frac{v_{i}}{w_{i+1}^{\min }} \rho_{i}+\rho_{m, i+1} \\
\overleftarrow{\mathrm{FC}}: \quad 0<\rho_{i} \leq \rho_{c, i}^{\min } \text { and } \\
& \rho_{m, i+1} \geq \rho_{i+1}>-\frac{v_{i}}{w_{i+1}^{\max }} \rho_{i}+\rho_{m, i+1} \\
\mathrm{CC}: & \rho_{c, i}^{\max }<\rho_{i} \leq \rho_{m, i} \text { and } \rho_{c, i+1}^{\max }<\rho_{i+1} \leq \rho_{m, i+1}
\end{array}
$$

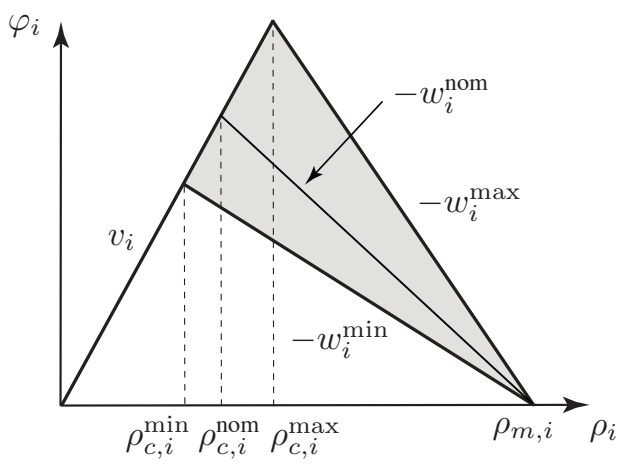

Fig. 4. Uncertain fundamental diagram: the speed of the congestion wave $w_{i}$ is not exactly known and ranges between $w_{i}^{\min }$ and $w_{i}^{\max }$. 


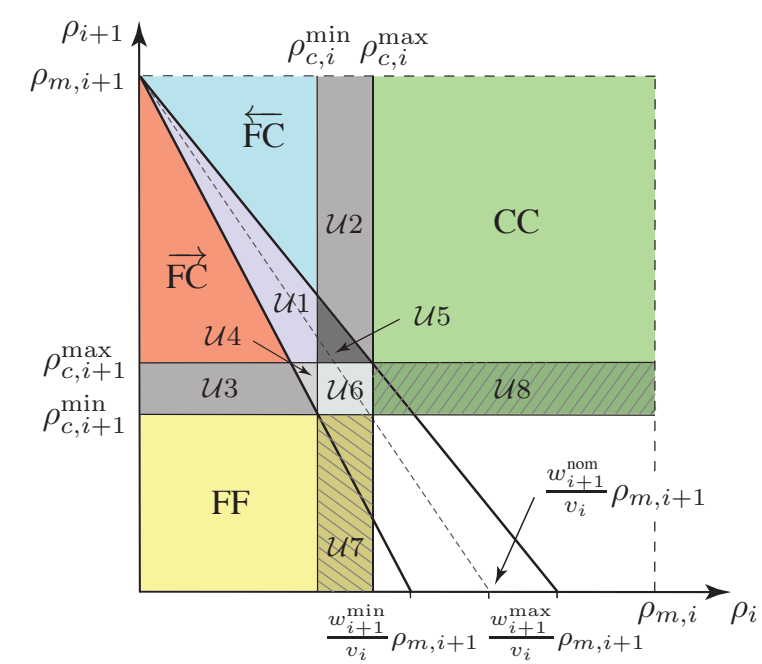

Fig. 5. Uncertain case: the speed of the congestion wave is not exactly known and we can identify four deterministic regions (colored) and six admissible uncertain regions $\mathcal{U} 1, \ldots, \mathcal{U} 6$ (shades of gray).

and eight uncertain regions (shades of gray in Fig. 5):

$\mathcal{U} 1, \overrightarrow{\mathrm{FC}}$ or $\overleftarrow{\mathrm{FC}}$ :

$$
-\frac{v_{i}}{w_{i+1}^{\text {min }}} \rho_{i}+\rho_{m, i+1}<\rho_{i+1} \leq-\frac{v_{i}}{w_{i+1}^{\text {max }}} \rho_{i}+\rho_{m, i+1}
$$

$\mathcal{U} 2, \overleftarrow{\mathrm{FC}}$ or $\mathrm{CC}$ :

$\rho_{c, i}^{\min }<\rho_{i} \leq \rho_{c, i}^{\max }$ and $\rho_{m, i+1} \geq \rho_{i+1}>-\frac{v_{i}}{w_{i+1}^{\text {max }}} \rho_{i}+\rho_{m, i+1}$

$\mathcal{U} 3, \overrightarrow{\mathrm{FC}}$ or $\mathrm{FF}$ :

$$
\rho_{i+1} \leq-\frac{v_{i}}{w_{i+1}^{\min }} \rho_{i}+\rho_{m, i+1} \text { and } \rho_{c, i+1}^{\min }<\rho_{i+1} \leq \rho_{c, i+1}^{\max }
$$

$\mathcal{U} 4$, FF or $\overleftarrow{\mathrm{FC}}$ :

$0<\rho_{i} \leq \rho_{c, i}^{\min }$ and $\rho_{c, i+1}^{\max } \geq \rho_{i+1}>-\frac{v_{i}}{w_{i+1}^{\min }} \rho_{i}+\rho_{m, i+1}$

$\mathcal{U} 5$, CC or $\overrightarrow{\mathrm{FC}}$ :

$$
\rho_{i} \geq \rho_{c, i}^{\min } \text { and } \rho_{c, i+1}^{\max }<\rho_{i+1} \leq-\frac{v_{i}}{w_{i+1}^{\max }} \rho_{i}+\rho_{m, i+1}
$$

$\mathcal{U} 6$, FF or $\mathrm{CC}$ :

$$
\rho_{c, i}^{\min }<\rho_{i} \leq \rho_{c, i}^{\max } \text { and } \rho_{c, i+1}^{\min }<\rho_{i+1} \leq \rho_{c, i+1}^{\max }
$$

Remark 1: Note that $\mathcal{U} 7$ and $\mathcal{U} 8$ in Fig. 5 can be actually regarded as deterministic regions since the mode $\mathrm{CF}$ is not admissible by hypothesis, and they can then be fused into the regions $\mathrm{FF}$ and $\mathrm{CC}$, respectively.

Note that if the state of the SMM lies in one of the uncertain regions $\mathcal{U} 1, \ldots, \mathcal{U} 6$ shown in Fig. 5, we need additional information to determine in which one of two possible modes the system currently is. Indeed, useful statistical information can be inferred from the historical data relative to the speed of the congestion wave. Next, we describe an algorithm to build a robust mode transition digraph $\mathscr{D}_{2}^{\text {rob }}$ which incorporates the information by historical record. Without loss of generality, we can restrict our analysis to region $\mathcal{U} 1$ where we shall compute the probability of being either in $\overrightarrow{\mathrm{FC}}$ or in $\overleftarrow{\mathrm{FC}}$.

Algorithm 1 (Disambiguation in region $\mathcal{U} 1$ ):

1) Consider cell $i$ and the flow vs. density data relative to a fixed historical record, consisting of multiple instances of the same day. Determine the point having maximum ordinate and split the data cloud using the value of its abscissa. Compute the historical free-flow speed and the nominal speed of the congestion wave $w_{i}^{\text {nom }}$ via (constrained) least-squares regression (the jam density is assigned, by hypothesis), from which the nominal critical density $\rho_{c, i}^{\text {nom }}$ can be determined.

2) Take the points $\left\{\left(\rho_{j}, \varphi_{j}\right)\right\}_{j=1}^{\ell}$ that lie in the congested side of the uncertain fundamental diagram and compute the set of historical speeds of congestion wave as $w_{i, j}=\frac{\varphi_{j}}{\rho_{m, i}-\rho_{j}}, j \in\{1, \ldots, \ell\}$.

3) Determine the speed variations with respect to the nominal value, $\Delta w_{i, j}=w_{i, j}-w_{i}^{\text {nom }}, j \in\{1, \ldots, \ell\}$.

4) Compute the empirical cumulative distribution function (CDF) $F\left(\Delta w_{i, j}\right)$ of $\Delta w_{i, j}, j \in\{1, \ldots, \ell\}$.

5) Approximate $F\left(\Delta w_{i, j}\right)$ with an arctangent function $F_{\text {app }}\left(\Delta w_{i, j}\right) \triangleq a \arctan \left(b \Delta w_{i, j}+c\right)+d$ where $a, b$, $c, d \in \mathbb{R}$ are parameters to be determined via nonlinear least-squares fitting.

6) Compute the median $F_{\text {app }}^{-1}(1 / 2) \triangleq \Delta w_{i}^{\text {med }}=$ $\frac{1}{b}\left[\tan \left(\frac{1 / 2-d}{a}\right)-c\right]$ from which one can get $w_{i}^{\text {med }}=$ $\Delta w_{i}^{\text {med }}+w_{i}^{\text {nom }}$ and $\rho_{c, i}^{\text {med }}=\frac{w_{i}^{\text {med }}}{v_{i}^{h}+w_{i}^{\text {med }}} \rho_{m, i}$.

Parameters $w_{i}^{\text {med }}, \rho_{c, i}^{\text {med }}$ can be used to modify the diagram in Fig. 5 as shown in Fig. 6(a). Fig. 6(b) reports the corresponding transition digraph $\mathscr{D}_{2}^{\text {Rob }}$ that we can utilize in our robust mode selector: $s(k)=\Sigma\left(\boldsymbol{\rho}(k), \boldsymbol{\varphi}(k), \mathscr{D}_{2}^{\mathrm{Rob}}\right)$.

\section{APPLICATION TO TRAFFIC DENSITY ESTIMATION}

Building upon [3], in this section the theory presented in Sect. III is applied to the problem of traffic density reconstruction via a switching observer, and validated using real data from Grenoble south ring.

\section{A. Robust estimation of traffic density}

Consider the following robust switching Luenberger observer of system (4):

$$
\left\{\begin{aligned}
& \widehat{\boldsymbol{\rho}}(k+1)=\mathbf{A}_{\hat{s}(k)} \widehat{\boldsymbol{\rho}}(k)+\mathbf{B}_{\hat{s}(k)} \boldsymbol{\varphi}(k)+\mathbf{E}_{\hat{s}(k)} \boldsymbol{\rho}_{m} \\
&+\mathbf{K}_{\hat{s}(k)}(y(k)-h(\widehat{\boldsymbol{\rho}}(k), \hat{s}(k))), \\
& \widehat{s}(k)=\Sigma\left(\widehat{\boldsymbol{\rho}}(k), \boldsymbol{\varphi}(k), \mathscr{D}_{2}^{\mathrm{Rob}}\right),
\end{aligned}\right.
$$

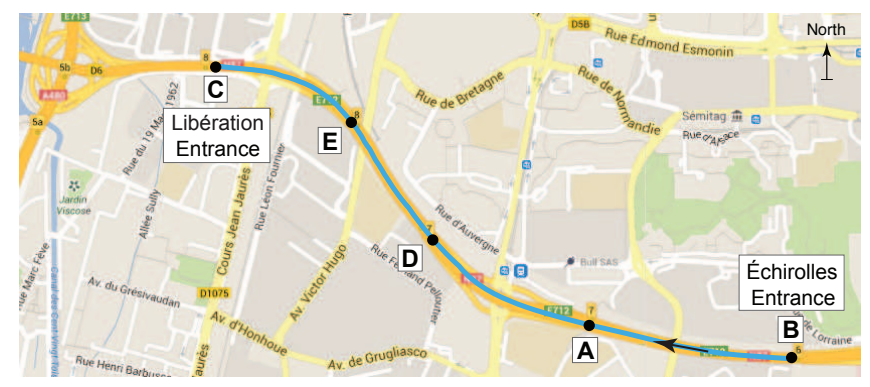

(a)

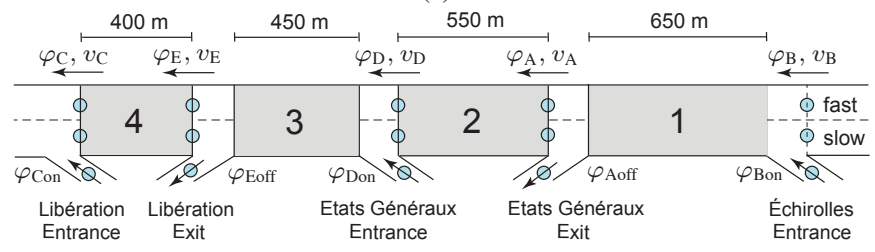

(b)

Fig. 7. (a) The section of Grenoble south ring considered in our experimental study (image source: Google Maps); (b) The section has been subdivided into four cells whose length ranges from 400 to 650 meters: the disks indicate pairs of magnetometers spaced $4.5 \mathrm{~m}$ apart. 


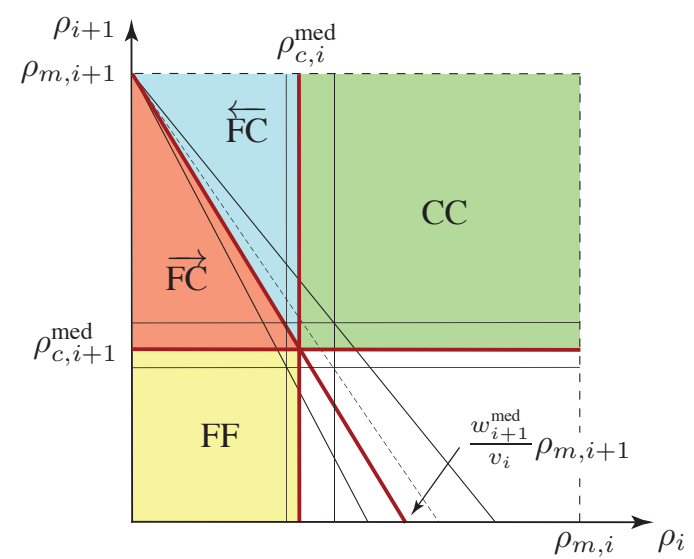

(a)

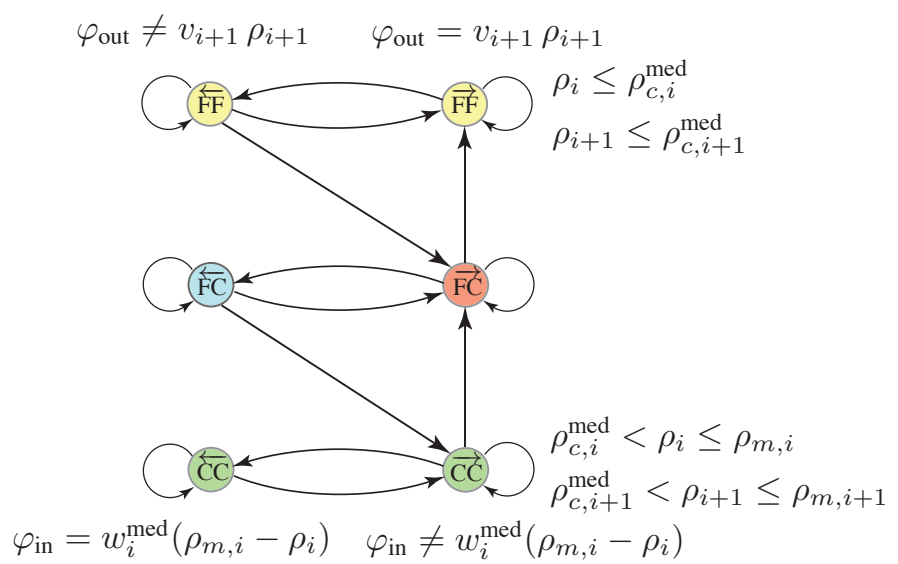

(b)

Fig. 6. (a) Modified mode representation in the $\rho_{i} \rho_{i+1}$-plane according to the historical record; (b) Corresponding mode-transition digraph $\mathscr{D}_{2}^{\text {Rob }}$ used in the robust selector $\Sigma\left(\boldsymbol{\rho}(k), \boldsymbol{\varphi}(k), \mathscr{D}_{2}^{\text {Rob }}\right)$ (the switching conditions for the central modes have been again omitted for improving readability).

where $\widehat{\boldsymbol{\rho}}=\left[\widehat{\rho}_{1}, \ldots, \widehat{\rho}_{n}\right]^{T}$ is the vector of density estimates, $\Sigma\left(\widehat{\rho}(k), \boldsymbol{\varphi}(k), \mathscr{D}_{2}^{\mathrm{Rob}}\right)$ is the robust mode selector which outputs the estimated mode $\widehat{s}(k) \in\{1, \ldots, M\}$, and $\mathbf{K}_{\hat{s}(k)}$ is the observer gain vector (which is nonzero only for modes 1 and $M$ ). Following [3], we can select $\mathbf{K}_{\hat{s}(k)}, \widehat{s}(k) \in\{1, M\}$, using a pole-placement method, such that $\operatorname{sprad}\left(\mathbf{A}_{\hat{s}(k)}-\mathbf{K}_{\hat{s}(k)} \mathbf{C}_{\hat{s}(k)}\right) \leq \lambda_{\min }\left(\mathbf{A}_{\hat{s}(k)}\right)<1$ where $\operatorname{sprad}(\cdot)$ and $\lambda_{\min }(\cdot)$ denote the spectral radius and smallest eigenvalue of a matrix, respectively. Note that in (5), $y(k)=\varphi_{d}(k)$ if $\widehat{s}(k)=1$ and $y(k)=\varphi_{u}(k)$ if $\widehat{s}(k)=M$, and that $\mathbf{A}_{\hat{s}(k)}=\mathbf{A}_{\hat{s}(k)}\left(\mathbf{v}, \mathbf{w}^{\text {med }}\right), \mathbf{C}_{M}=\mathbf{C}_{M}\left(\mathbf{w}^{\text {med }}\right)$, $\mathbf{E}_{\hat{s}(k)}=\mathbf{E}_{\hat{s}(k)}\left(\mathbf{w}^{\text {med }}\right)$, where $\mathbf{w}^{\text {med }} \triangleq\left[w_{1}^{\text {med }}, \ldots, w_{n}^{\text {med }}\right]^{T}$ and $\mathbf{v} \triangleq\left[v_{1}, \ldots, v_{n}\right]^{T}$, cf. Sec. III-B.

\section{B. Experimental validation}

The performance of the robust density estimator (5) has been tested with real traffic data coming from a section
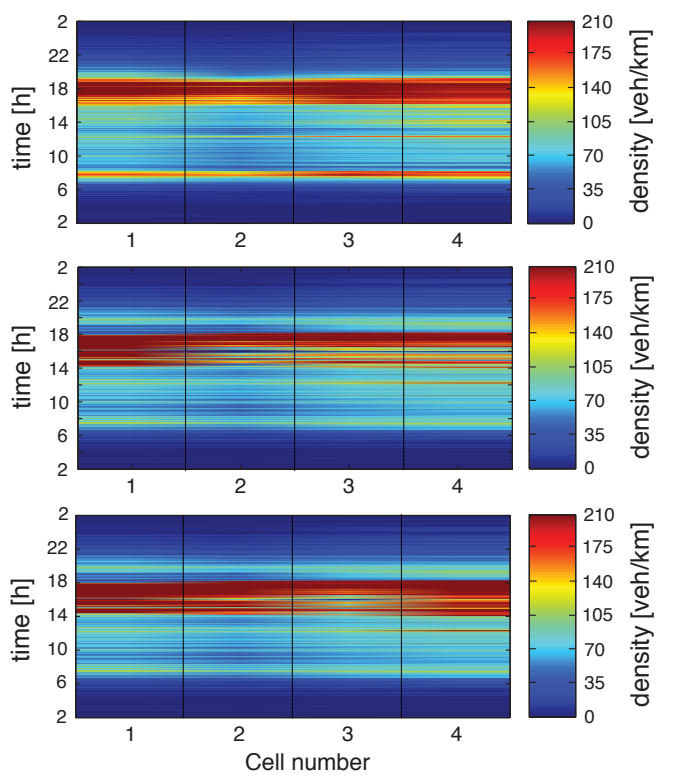

Fig. 8. Experimental results for Tuesday, June 25, 2013: Density reconstruction in the four cells: (top) ground truth; (middle) observer in [3]; (bottom) robust observer (5). of $2.2 \mathrm{~km}$ in the west end of Grenoble south ring, a highway enclosing the southern part of the city from A41 to A480. This two-lane section stretches westward from "Échirolles Entrance" to "Libération Entrance", includes 3 on-ramps and 2 off-ramps (see Fig. 7(a)), and it is equipped with 3 pairs of Sensys Networks VDS240 wireless magneto-resistive sensors embedded in the pavement along the fast/slow lanes and on-/off-ramps at locations B, A, D, E and C (see Fig. 7(b)). The sensors provide flow and time-mean speed measurements every $15 \mathrm{~s}$ : the data from the fast and slow lanes were combined to yield single mainline information $\left(\varphi_{\mathrm{B}}, \varphi_{\mathrm{A}}, \ldots, \varphi_{\mathrm{E}}\right.$ and $\left.v_{\mathrm{B}}, v_{\mathrm{A}}, \ldots, v_{\mathrm{E}}\right)$. This section of the south ring was chosen since a significant level of congestion emanating from point $\mathrm{C}$ and propagating backward can be observed in the weekdays during the morning $(7: 30-8: 15 \mathrm{~h})$ and evening (17:00-19:00h) rush hours: the speed limit is $90 \mathrm{~km} / \mathrm{h}$ at B, A, D, E, and $70 \mathrm{~km} / \mathrm{h}$ at C. The segment was subdivided into four cells whose length is $L_{1}=650 \mathrm{~m}$, $L_{2}=550 \mathrm{~m}, L_{3}=450 \mathrm{~m}$ and $L_{4}=400 \mathrm{~m} .24 \mathrm{~h}$ traffic data (starting at 2:00 in the morning) collected on Tuesday June 25, 2013 was utilized in our test. The speed data has been first corrected by filling possible gaps due to communication losses (via interpolation), and then the speed and flow data have been resampled to 1 and 1/2 min and filtered with a 1st-order low-pass Butterworth filter with normalized cutoff frequency 0.05 . The erratic behavior of the mainline detectors at $B$ forced us to infer their flow measurements from the corresponding detectors at $A$ : this yielded a percentage of vehicle losses between $B$ and $C$ of about $0.6 \%$. The highway section was modeled as (see Fig. 7(b)):

$$
\begin{aligned}
\rho_{1}(k+1) & =\rho_{1}(k)+\frac{T}{L_{1}}\left(\varphi_{\mathrm{B}}(k)+\varphi_{\mathrm{Bon}}(k)\right. \\
& \left.-\min \left\{D_{1}, S_{2}+\varphi_{\text {Aoff }}(k)\right\}\right), \\
\rho_{2}(k+1) & =\rho_{2}(k)+\frac{T}{L_{2}}\left(\min \left\{D_{1}-\varphi_{\text {Aoff }}(k), S_{2}\right\}\right. \\
& \left.-\min \left\{D_{2}, S_{3}-\varphi_{\text {Don }}(k)\right\}\right), \\
\rho_{3}(k+1) & =\rho_{3}(k)+\frac{T}{L_{3}}\left(\min \left\{D_{2}+\varphi_{\text {Don }}(k), S_{3}\right\}\right. \\
& \left.-\min \left\{D_{3}, S_{4}+\varphi_{\text {Eoff }}(k)\right\}\right), \\
\rho_{4}(k+1) & =\rho_{4}(k)+\frac{T}{L_{4}}\left(\min \left\{D_{3}-\varphi_{\text {Eoff }}(k), S_{4}\right\}-\varphi_{\mathrm{C}}(k)\right),
\end{aligned}
$$

with $D_{i-1}, S_{i}, i \in\{2,3,4\}$ defined in (2), yielding $\varphi=\left[\varphi_{\mathrm{B}}, \varphi_{\text {Bon }}, \varphi_{\text {Aoff }}, \varphi_{\text {Don }}, \varphi_{\text {Eoff }}, \varphi_{\mathrm{C}}\right]^{T}$ and $M=10$ 


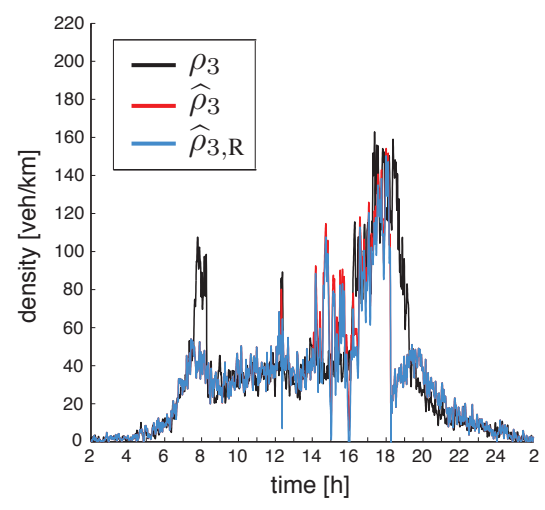

(a)

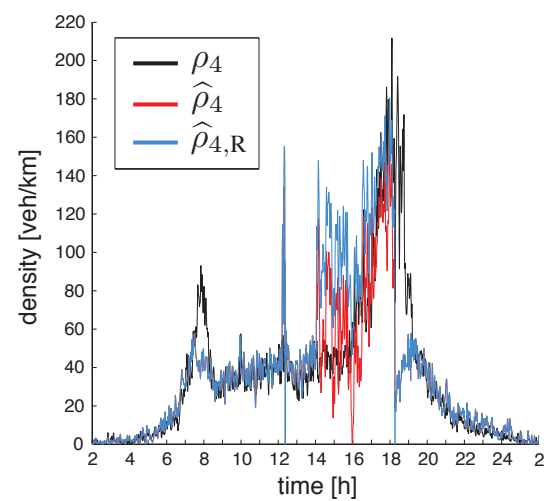

(b)
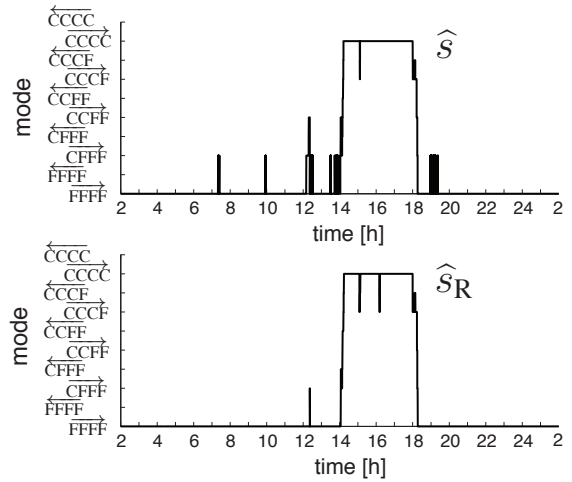

(c)

Fig. 9. Experimental results for Tuesday, June 25, 2013: (a)-(b) Density profiles of cells 3 and 4 reconstructed by the observer in [3] (red) and by the robust observer (5) (blue, subscript "R") against the ground truth (black); (c) Sequence of modes estimated by the observer in [3] (top) and by (5) (bottom).

for the observer in (5) with $n=4$. We set $T=$ $15 / 3600 \mathrm{~h}, \boldsymbol{\rho}(0)=[0,0,0,0]^{T}$, and imposed the following eigenvalues of $\mathbf{A}_{\hat{s}}-\mathbf{K}_{\hat{s}} \mathbf{C}_{\hat{s}}$, $\widehat{s} \in\{1,10\}$, for the observer (5) and the observer in [3]: $\{0.05,0.1,0.15,0.2\}$ and $\{0.6,0.65,0.7,0.75\}$. An automatic procedure (which only requires the user to provide the jam densities $\rho_{m, 1}, \ldots, \rho_{m, 4}$, fixed to $250 \mathrm{veh} / \mathrm{km}$ in this study), has been devised to calibrate the parameters of system (6). It yielded the following free-flow speeds: $v_{1}=83.52, v_{2}=84.28, v_{3}=79.84$, $v_{4}=71.36 \mathrm{~km} / \mathrm{h}$. Moreover, $w_{1}^{\text {med }}=24.20, \rho_{c, 1}^{\text {med }}=56.12$, $w_{2}^{\text {med }}=19.32, \rho_{c, 2}^{\text {med }}=52.28, w_{3}^{\text {med }}=18.58, \rho_{c, 3}^{\text {med }}=47.56$, $w_{4}^{\text {med }}=21.82, \rho_{c, 4}^{\text {med }}=66.94$ (in $\mathrm{km} / \mathrm{h}$ and veh $/ \mathrm{km}$ ), which were computed from the data of four historical Tuesdays (May 29, June 5, 12, 18 of 2013) using Algorithm 1. Fig. 8 reports the "measured densities" for the four cells (top), i.e. the densities directly reconstructed from the flow and mean speed measurements (our ground truth), and the densities estimated by the observer in [3] (middle) and by the robust estimator (5) (bottom). From the figure, we can note that the proposed observer is able to more accurately capture the evening congestion. Figs. 9(a)-(b) show in greater detail the time profiles of the densities of cells 3 and 4 estimated by the observer in [3] (red) and by the robust observer (5) (blue, subscript "R"), against the ground truth (black). The density root-mean-square deviation for the robust estimator, $\operatorname{RMSD}_{i}=\left(\frac{1}{\mathrm{~N}} \sum_{k=0}^{\mathrm{N}-1}\left(\rho_{i}(k)-\widehat{\rho}_{i, \mathrm{R}}(k)\right)^{2}\right)^{1 / 2}, i \in\{1,2,3,4\}$, $\mathrm{N}=5760$, is $46.44,27.74,24.16$ and $24.11 \mathrm{veh} / \mathrm{km}$. Finally, Fig. 9(c) reports the sequence of modes estimated by the observer in [3] (top, $\widehat{s}$ ) and by (5) (bottom, $\widehat{s}_{\mathrm{R}}$ ), from which we can notice that the robust estimator switches between different modes less frequently than the observer in [3], ultimately leading to steadier and more reliable traffic density estimates.

\section{CONCLUSIONS AND FUTURE WORK}

In this paper we have introduced a new robust mode selector for the uncertain graph-constrained SMM which we have applied to the problem of highway traffic density estimation via a switching observer. The selector leverages a suitably-defined transition digraph and makes a judicious use of the currently-available and historical traffic information in order to identify the most probable mode of the SMM. Experimental tests with traffic data from a $2.2 \mathrm{~km}$ section of Grenoble south ring have supported our theoretical findings.
In this work, we have only dealt with an uncertain speed of the congestion wave: in the future, we aim at including other sources of uncertainty in the SMM, such as uncertain initial densities and boundary flows, and at providing guaranteed bounds on the traffic density evolution. The possibility to formulate our density-estimation problem in the framework of Markov jump linear systems as in [4], and the validation of our robust observer on the overall $10.5 \mathrm{~km}$ Grenoble south ring, are other subjects of on-going research.

\section{REFERENCES}

[1] C.F. Daganzo. The cell transmission model: a dynamic representation of highway traffic consistent with the hydrodynamic theory. Transport. Res. B-Meth., 4(28):269-287, 1994.

[2] L. Mun̄oz, X. Sun, R. Horowitz, and L. Alvarez. Traffic density estimation with the cell transmission model. In Proc. American Contr. Conf, pages 3750-3755, 2003.

[3] C. Canudas de Wit, L. León Ojeda, and A.Y. Kibangou. Graph Constrained-CTM observer design for the Grenoble south ring. In Proc. 13rd IFAC Symp. Contr. Transp. Syst., pages 197-202, 2012.

[4] X. Sun, L. Muñoz, and R. Horowitz. Highway traffic state estimation using improved mixture Kalman filters for effective ramp metering control. In Proc. 42nd IEEE Conf. Dec. Contr, volume 6, pages 63336338, 2003.

[5] A. Lemarchand, J.J. Martinez, and D. Koenig. Smooth Switching $\mathrm{H}_{\infty}$ PI Controller for Local Traffic On-ramp Metering, an LMI Approach. In Proc. 18th IFAC World Congr., pages 13882-13887, 2011.

[6] A.A. Kurzhanskiy and P. Varaiya. Guaranteed prediction and estimation of the state of a road network. Transport. Res. C-Emer, 21(1):163$180,2012$.

[7] S. Blandin, D. Work, P. Goatin, B. Piccoli, and A. Bayen. A general phase transition model for vehicular traffic. SIAM J. Appl. Math. 71(1):107-127, 2011

[8] A. Nún̄ez and B. De Schutter. Distributed Identification of Fuzzy Confidence Intervals for Traffic Measurements. In Proc. 51st IEEE Conf. Dec. Contr, pages 6995-7000, 2012.

[9] Y. Wang and M. Papageorgiou. Real-time freeway traffic state estimation based on extended Kalman filter: a general approach. Transport. Res. B-Meth, 39:141-167, 2005.

[10] C.M.J. Tampère and L.H. Immers. An extended Kalman filter application for traffic state estimation using CTM with implicit mode switching and dynamic parameters. In Proc. IEEE Int. Transp. Syst. Conf., pages 209-216, 2007.

[11] A. Hegyi, D. Girimonte, R. Babuška, and B. De Schutter. A comparison of filter configurations for freeway traffic state estimation. In Proc. IEEE Int. Transp. Syst. Conf., pages 1029-1034, 2006.

[12] L. Mihaylova, R. Boel, and A. Hegyi. Freeway traffic estimation within particle filtering framework. Automatica, 43:290-300, 2007.

[13] A. Sumalee, R.X. Zhong, T.L. Pan, and W.Y. Szeto. Stochastic cell transmission model (SCTM): A stochastic dynamic traffic model for traffic state surveillance and assignment. Transport. Res. B-Meth., 45(3):507-533, 2011.

[14] I.-C. Morarescu and C. Canudas de Wit. Highway traffic model-based density estimation. In Proc. American Contr. Conf, pages 2012-2017, 2011. 